\title{
What We Learn from the X-Ray Grating Spectra of Nova SMC 2016
}

\author{
M. Orio ${ }^{1,2}$ (1) J.-U. Ness ${ }^{3}$, A. Dobrotka ${ }^{4}$, E. Gatuzz ${ }^{5}$, N. Ospina ${ }^{6}$, E. Aydi ${ }^{7}$, E. Behar ${ }^{8,9}$, D. A. H. Buckley ${ }^{7}$, S. Ciroi ${ }^{10}$,

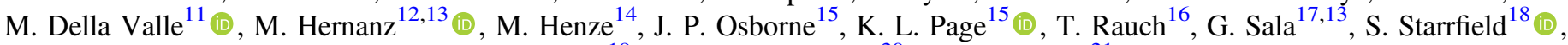 \\ R. E. Williams ${ }^{19}$ (D) C. E. Woodward ${ }^{20}$, and P. Zemko ${ }^{21}$ (D) \\ ${ }^{1}$ Department of Astronomy, University of Wisconsin, 475 N. Charter St., Madison WI 53706, USA; orio@ astro.wisc.edu \\ 2 INAF-Osservatorio Astronomico di Padova, vicolo Osservatorio, 5, I-35122 Padova, Italy \\ ${ }^{3}$ XMM-Newton Science Operations Center, European Space Astronomy Center, Camino Bajo del Castillo s/n, Urb. Villafranca del Castillo, Villanueva de la Caada, \\ E-28692 Madrid, Spain \\ ${ }^{4}$ Advanced Technologies Research Institute, Faculty of Materials Science and Technology in Trnava, Slovak University of Technology in Bratislava, Bottova 25, 917 \\ 24 Trnava, Slovakia \\ ${ }^{5}$ ESO, Karl-Schwarzschild-Strasse 2, D-85748 Garching bei München, Germany \\ ${ }^{6}$ via Pietro Pomponazzi, 33, I-35124 Padova, Italy \\ ${ }^{7}$ South African Astronomical Observatory, P.O. Box 9, 7935 Observatory, South Africa \\ ${ }^{8}$ Department of Physics, Technion, Haifa, Israel \\ ${ }^{9}$ Astronomy Department, University of Cape Town, 7701 Rondebosch, South Africa \\ ${ }^{10}$ Dipartimento di Fisica e Astronomia, vicolo Osservatorio, 3, I-35122 Padova, Italy \\ ${ }^{11}$ INAF-Osservatorio Astronomico di Capodimonte, Salita Moiarello, 16, I-80131 Napoli, Italy \\ ${ }^{12}$ Institut de Ciències de l' Espai (ICE-CSIC), Campus UAB, c/ Can Magrans s/n, E-08193, Bellaterra, Spain \\ ${ }^{13}$ Institut d'Estudis Espacials de Catalunya, c/Gran Capità 2-4, Ed. Nexus-201, E-08034, Barcelona, Spain \\ ${ }^{14}$ Department of Astronomy, San Diego State University, San Diego, CA 92182, USA \\ ${ }^{15}$ X-ray and Observational Astronomy Group, Department of Physics \& Astronomy, University of Leicester, LE1 7RH, UK \\ ${ }^{16}$ Institute for Astronomy and Astrophysics, Kepler Center for Astro and Particle Physics, Eberhard Karls University, Sand.1., D-72076 Tübingen, Germany \\ ${ }^{17}$ Departament de Física, EEBE, Universitat Politècnica de Catalunya, BarcelonaTech., Av. d' Eduard Maristany 10-14, E-08019, Barcelona, Spain \\ ${ }^{18}$ School of Earth and Space Exploration, Arizona State University, Tempe, AZ 85287-1404, USA \\ ${ }^{19}$ Space Telescope Science Institute, 3700 San Martin Drive, Baltimore, MD 21218 USA \\ ${ }^{20}$ Minnesota Institute for Astrophysics, University of Minnesota, 116 Church Street, SE, Minneapolis, MN 55455, USA \\ ${ }^{21}$ via dei Tadi, 5, I-35139 Padova, Italy \\ Received 2018 May 3; revised 2018 June 21; accepted 2018 June 22; published 2018 August 3
}

\begin{abstract}
Nova SMC 2016 has been the most luminous nova known in the direction of the Magellanic Clouds. It turned into a very luminous supersoft X-ray source between days 16 and 28 after the optical maximum. We observed it with Chandra, the HRC-S camera, and the Low Energy Transmission Grating on 2016 November and 2017 January (days 39 and 88 after optical maximum), and with XMM-Newton on 2016 December (day 75). We detected the compact white dwarf (WD) spectrum as a luminous supersoft X-ray continuum with deep absorption features of carbon, nitrogen, magnesium, calcium, probably argon, and sulfur on day 39, and oxygen, nitrogen, and carbon on days 75 and 88. The spectral features attributed to the WD atmosphere are all blueshifted, by about $1800 \mathrm{~km} \mathrm{~s}^{-1}$ on day 39 and up to $2100 \mathrm{~km} \mathrm{~s}^{-1}$ in the following observations. Spectral lines attributed to low-ionization potential transitions in the interstellar medium are also observed. Assuming the distance to the Small Magellanic Cloud, the bolometric luminosity exceeded the Eddington level for at least three months. A preliminary analysis with atmospheric models indicates an effective temperature of around 700,000 K on day 39, peaking at the later dates in the $850,000-900,000 \mathrm{~K}$ range, as expected for $\mathrm{a} \simeq 1.25 M_{\odot}$ WD. We suggest a possible classification as an oxygen-neon WD, but more precise modeling is needed to accurately determine the abundances. The X-ray light curves show a large, aperiodic flux variability, which is not associated with spectral variability. We detected red noise, but did not find periodic or quasiperiodic modulations.
\end{abstract}

Key words: novae, cataclysmic variables - stars: abundances - stars: dwarf novae - stars: individual (N SMC 2016a) - X-rays: stars

\section{Introduction}

Novae in outburst are among the most luminous stellar X-ray sources in the sky. A recent overview of the observational facts has been given by Poggiani (2018). Comprehensive reviews of the models can be found in Starrfield et al. $(2016,2012)$ and Prialnik \& Kovetz (2005), and the basic facts can be summarized as follows. Nova eruptions are due to thermonuclear burning of hydrogen via the CNO cycle, at the bottom of a shell accreted by a white dwarf (WD) from a close binary companion. The outburst is repeated after quiescent periods ranging from a few years to $\simeq$ hundreds of thousands years. The burning is ignited in conditions of electron degeneracy and becomes explosive, inflating and possibly immediately ejecting part of the envelope. Since the initial suggestion of Bath \& Shaviv (1976), many authors working on models have predicted that the bulk of the remaining envelope mass is then stripped by a radiation-pressure-driven wind, although a wind may also be triggered by Roche Lobe overflow (see, for a discussion, Wolf et al. 2013). Then, the evolutionary track of the post-nova is driven by a shift in the wavelength of the maximum energy toward shorter wavelengths, at a constant bolometric luminosity close to $10^{38} \mathrm{erg} \mathrm{s}^{-1}$ (e.g., Starrfield et al. 2012; Wolf et al. 2013). This phase lasts from one week to $\approx 10$ years as the WD photosphere shrinks close to preoutburst radius, while thermonuclear burning is still occurring near the surface. Thus, post-outburst novae offer a unique possibility to observe the effects of nuclear burning near the 
Table 1

Chandra and XMM-Newton Observations of the Nova SMC 2016 Examined in this Article, and the Measured Count Rates for the X-ray Detectors

\begin{tabular}{|c|c|c|c|c|c|c|}
\hline Instrument & Obs ID & $\begin{array}{l}\text { Exp. Time } \\
\text { (ks) }\end{array}$ & Date $^{\mathrm{b}}$ & Day $^{\mathrm{c}}$ & $\begin{array}{c}\text { c.r. } \\
\text { counts s }^{-1}\end{array}$ & $\begin{array}{c}L_{\mathrm{X}}(60 \mathrm{kpc})^{\mathrm{d}} \\
\mathrm{erg} \mathrm{s}^{-1}\end{array}$ \\
\hline Chandra HRC-S+LETG & 19011 & 30.16 & 2016 Nov 17 & 39 & $6.589 \pm 0.011$ & $3.42 \times 10^{38}$ \\
\hline XMM-Newton RGS & 079418020 & 29.80 & 2016 Dec 22 & 75 & $24.56 \pm 0.02$ & $3.9 \times 10^{38}$ \\
\hline
\end{tabular}

Notes.

${ }^{\text {a }}$ Exposure time of the observation (dead-time corrected).

b Start date of the observation.

c Time in days after the discovery on 2016 October 14.

d In the 10-60 ̊ range for Chandra, in the 10-38 $\AA$ range for the XMM-Newton RGS.

stellar surface. The WD reaches an effective temperature $T_{\text {eff }}>200,000 \mathrm{~K}$, emitting in the X-ray range (Starrfield et al. 2012; Wolf et al. 2013), and becomes a supersoft X-ray source (SSS). Despite its high luminosity, the X-ray flux of the burning WD is very easily absorbed. If the column density exceeds $10^{22} \mathrm{~cm}^{-2}$, it may never be detected. In most cases, however, the column density and/or the filling factor of the ejecta must be sufficiently low that the WD becomes observable in X-rays at large distances, at the outskirts of the Local Group and beyond, wherever the interstellar column density is low (see Orio 2012; Henze et al. 2014 and references therein).

There is a second source of X-ray flux in novae in outburst, with different timescale and evolution in each nova: it is the $\mathrm{X}$-ray emission that originates early in the outburst from the ejecta, most likely because of violent shocks in colliding winds (Orio 2012 and references therein). In most novae, the peak luminosity of this emission from the shell is $10^{34} \mathrm{erg} \mathrm{s}^{-1}$ (see, e.g., Orio et al. 1996, 2001; Peretz et al. 2016). In symbiotic novae, in which the secondary is a red giant, the impact of the ejecta with the giant wind produces thermal $\mathrm{X}$-ray emission with a peak luminosity even as high as $10^{36} \mathrm{erg} \mathrm{s}^{-1}$ (Ness et al. 2007; Nelson et al. 2008). The initial temperature of the ejecta exceeds $10 \mathrm{keV}$, but the shocked plasma is observed to cool with time until the emission lines are only in the very soft range. Often, the soft X-rays' emission lines are superimposed on the spectrum of the central source (see Orio 2012, Orio et al. 2013, and references therein).

Novae in the Magellanic Clouds are sufficiently close to us and in directions of such low column density that we can obtain $\mathrm{X}$-ray grating spectra to study them in detail; they offer us the opportunity to study targets at a known distance and to compare the population of Galactic novae with one in a much lower metallicity environment.

\section{An Intriguingly "Hyperluminous" Nova}

A comprehensive description of the observational facts concerning N SMC 2016 can be found in Aydi et al. (2018), so we will give here only a brief summary. The nova was discovered by MASTER (as OT J010603.18-744715.8) on 2016 October 14 (Shumkov et al. 2016), but the outburst had started earlier, with a maximum magnitude detected on 2016 October 9 (Jablonski \& Oliveira 2016; Lipunov et al. 2016). The latter is the date from which we count the post-outburst days in our list of observations in Table 1. N SMC 2016 was a very fast nova according to the classification by Warner (2003), with the time for decline by two visual magnitudes $t_{2}=4 \pm 1.0$ days, an FWHM of the Balmer lines of

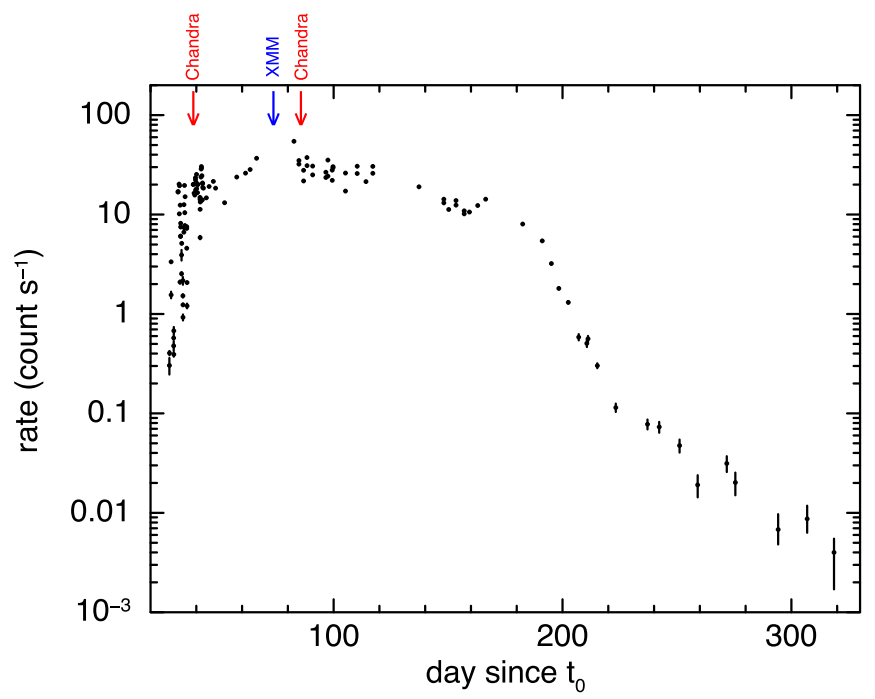

Figure 1. The Swift XRT light curve in the $0.3-10 \mathrm{keV}$ range published by Aydi et al. (2018); the dates of observations with Chandra and XMM-Newton are indicated. The initial time of optical maximum was 2016 October 9.

$3500 \pm 100 \mathrm{~km} \mathrm{~s}^{-1}$, and an amplitude of $12.1 \pm 1$ visual magnitudes.

The most striking characteristic of this nova is its extreme peak luminosity, larger than any known nova in the Magellanic Clouds. In the MASTER Very Wide Field Camera, which measures visual magnitude almost exactly corresponding to Johnson $V$ (unless the $\mathrm{H} \alpha$ line was already prominent, skewing the spectral energy distribution, but this would be unusual already at maximum), the nova reached $V=8.9 \pm 0.3$ on HJD 2457671.3 on 2016 October 9 (note that the value given by Lipunov et al. 2016 has been corrected by Lipunov, in a private communication to us). If we assume an average distance modulus of 18.96 (Scowcroft et al. 2016; corresponding to $62 \mathrm{kpc}$ ) and only moderate reddening, we find an absolute magnitude $V \simeq-10.1$, about 11 times the Eddington luminosity for a $1 M_{\odot}$ star. The optical and UV light curves presented in Aydi et al. (2018) imply super-Eddington luminosity for at least 88 days, but the Swift X-Ray Telescope (XRT) light curve analyzed in that article, which we show again here in Figure 1 to illustrate at what stages our observations were obtained, indicates that in the SSS stage, the X-ray luminosity (representative of most of the bolometric luminosity) did not decline significantly for about six months. As discussed by Aydi et al. (2018), the Small Magellanic Cloud (SMC) has a certain extension along the line of sight, so the distance modulus could be as low as 18.7 if the nova is on the side 
closer to us, but the luminosity would still be largely superEddington. In order to check the distance to verify whether the prolonged super-Eddington period was real, Aydi et al. (2018) evaluated instead the maximum absolute magnitude with the maximum magnitude versus rate of decline (MMRD) method and other empiric formulations, and concluded that the resulting distance would be only $d \simeq 42_{-7}^{+8} \mathrm{kpc}$, which would put the nova in front of the galaxy. However, this seems unlikely, so Aydi et al. (2018) still favored the SMC distance. Della Valle (1991) already found back in 1991 that the few very intrinsically luminous and fast novae, like N SMC 2016, do not follow the MMRD, so this may explain the non-SMC distance obtained when we try and apply the MMRD to Nova SMC 2016.

In fact, assuming that this nova is in the SMC, very few novae have been as intrinsically luminous at maximum as $\mathrm{N}$ SMC 2016. The Galactic nova CP Pup had a comparable peak absolute magnitude; another Galactic nova, V1500 Cyg (see Shafter et al. 2009, Strope et al. 2010, and references therein), and at least two extragalactic novae (M31N2007-11d and LMC 1991; see Schwarz et al. 2001) had maximum absolute magnitude $V \leqslant-9$. The parallax has been measured precisely with Gaia for CP Pup (for the conversion of parallax to distance, see Bailer-Jones et al. 2018 and Luri et al. 2018), and its large luminosity, around maximum absolute magnitude, around $V=-10$, has been confirmed. There seems to be also a distinct small population of superluminous novae in M31, the majority of which show Fe II type spectra and are fast, with $t_{2}<10$ days (S. C. Williams, presentation at EWASS2018 conference). N SMC 2016 is thus likely to belong to a rare class of superluminous novae, observed in the Galaxy and Magellanic Clouds only once in $\simeq 25$ years.

Aydi et al. (2018) discussed how the derived parameters point to a massive WD, about $1.25 M_{\odot}$ when compared with theoretical models by Wolf et al. (2013) and Hillman et al. (2016).

\section{The X-Ray Evolution}

Figure 1 shows the X-ray light curve measured with the Swift XRT (Burrows et al. 2005), already presented by Aydi et al. (2018). The nova was not detected in the first two weeks after the outburst. On 2016 October 26, it became unobservable with the XRT for 11 days due to the pole constraint (which means that Swift cannot point too close to the Earth limb), then on 2016 November 7, a luminous SSS was detected. Dense monitoring followed, initially three times per day, then twice a day, with another period of non-observability in the last two weeks of December. The X-ray source was variable, but the average X-ray luminosity was clearly increasing until about day 40 , followed by almost a plateau with an apparent peak around day 83 , when the luminosity started decreasing very slowly. A rapid decay started only around day 180 after the outburst. As Table 1 shows, a high-resolution X-ray spectrum was first taken on 2016 November 17 (day 39), shortly after the SSS discovery (with Chandra, using the HRC-S camera and the Low Energy Transmission Grating or LETG), and a few days before the maximum recorded count rate. It was observed again a few days after the maximum X-ray count rate on 2016 December 22 (with XMM-Newton, day 75) and again on 2017 January 4 (with the Chandra HRC-S+LETG, day 88). The Chandra HRC-S+LETG wavelength range is $1.2-175 \AA$ (corresponding to the energy range $0.07-10 \mathrm{keV}$ ), with a resolving power $\approx 20 \times \lambda$ in the $3-50 \AA$ range (and $\lambda / \Delta \lambda>1000$ at $50-160 \AA$ ), effective area peaking at $25 \mathrm{~cm}^{-2}$, and a resolution of $0.05 \AA$. The XMM-Newton RGS1 and RGS2 gratings probe the 5-38 $\AA$ spectral range (corresponding to $0.35-2.5 \mathrm{keV}$ ) with a maximum effective area of $61 \mathrm{~cm}^{2}$ at $15 \AA$, a wavelength accuracy of $5 \mathrm{m \AA}$, and bin size between 7 and $14 \mathrm{m \AA}$ in the first order. During the XMM-Newton exposures, all instruments were operated, including the Reflection Grating Spectrometers (RGS), in addition to the EPIC-pn and MOS cameras and the Optical Monitor (OM). The EPIC-pn camera calibrated energy range is $0.15-10 \mathrm{keV}$ and the EPIC-MOS range is $0.3-10 \mathrm{keV}$; because of the large pile-up in the EPIC data, for this paper we made use only of the RGS X-ray data. The OM data were obtained with the $U V W 2$ filter, which has an effective wavelength of $2120 \AA$ and a width of $500 \AA$. The nova was observed in fast mode (yielding a count rate measurement every $0.5 \mathrm{~s}$ ), in addition to the imaging mode that allows the magnitude integrating over much longer times to be calculated. We could not directly compare the magnitudes that were measured with the $U V W 2$ filter with the Swift UVOT light curve presented in Aydi et al. (2018), because the UVOT exposures were done only in a different bandpass filter at the time of the OM observation, but we find that the $\mathrm{OM}$ magnitudes are consistent with the trend of the UVOT light curve. We used the UV OM light curve in fast mode for the timing analysis.

\section{The High-resolution X-Ray Spectra}

We extracted the Chandra HRC-S+LETG spectra with their first-order grating redistribution matrix files and ancillary response files with the CIAO 4.9 task chandra repro, with version 4.7 of the calibration package CALDB. We coadded the positive- and negative-order spectra with "combine grating spectra" to increase the signal-to-noise ratio $(\mathrm{S} / \mathrm{N})$. We did not find it necessary to correct for the higher spectral orders, which gave negligible contamination.

We used the XMM-SAS software version 16.1.0 and ran the pipeline with the rgsproc task to obtain the spectra up to third order, but found only the +1 and -1 order spectra to be of interest. We combined them with rgscombine to obtain a higher $\mathrm{S} / \mathrm{N}$ spectrum.

The X-ray grating spectra are shown in Figure 2 and in more detail in Figure 3, in which we have marked the strongest absorption lines. We find absorption lines due to transitions of nitrogen and carbon in all three spectra, absorption lines of silicon, magnesium, calcium, and possibly argon and sulfur on day 39, and additionally, we detect $\mathrm{H}$-like and He-like oxygen features in the spectra of days 75 and 88 (indicating higher $\left.T_{\text {eff }}\right)$. All of the absorption lines that are consistent with the WD atmospheric origin are blueshifted by about $1800 \mathrm{~km} \mathrm{~s}^{-1}$ in the first observation, while the blueshift seems to vary a little for different lines and reaches up to $2100 \mathrm{~km} \mathrm{~s}^{-1}$ in the second and third observations. We added a question mark to a line of S XII (rest wavelength $30.514 \AA$ ) because there is also the possibility that it is instead Ca XI (rest wavelength $30.503 \AA$, but a stronger Ca XII at rest wavelength $30.448 \AA$ should fall at $30.266 \AA$ and is not observed). A line that overlaps and may even be blended with other features is the one attributed to $\operatorname{MgX}$ (rest wavelength $36.518 \AA$ ): it overlaps with a transition due to Ar XI and is also very near a $\mathrm{Cl}$ XI line (rest wavelength $36.518 \AA$ ). In order to finalize 


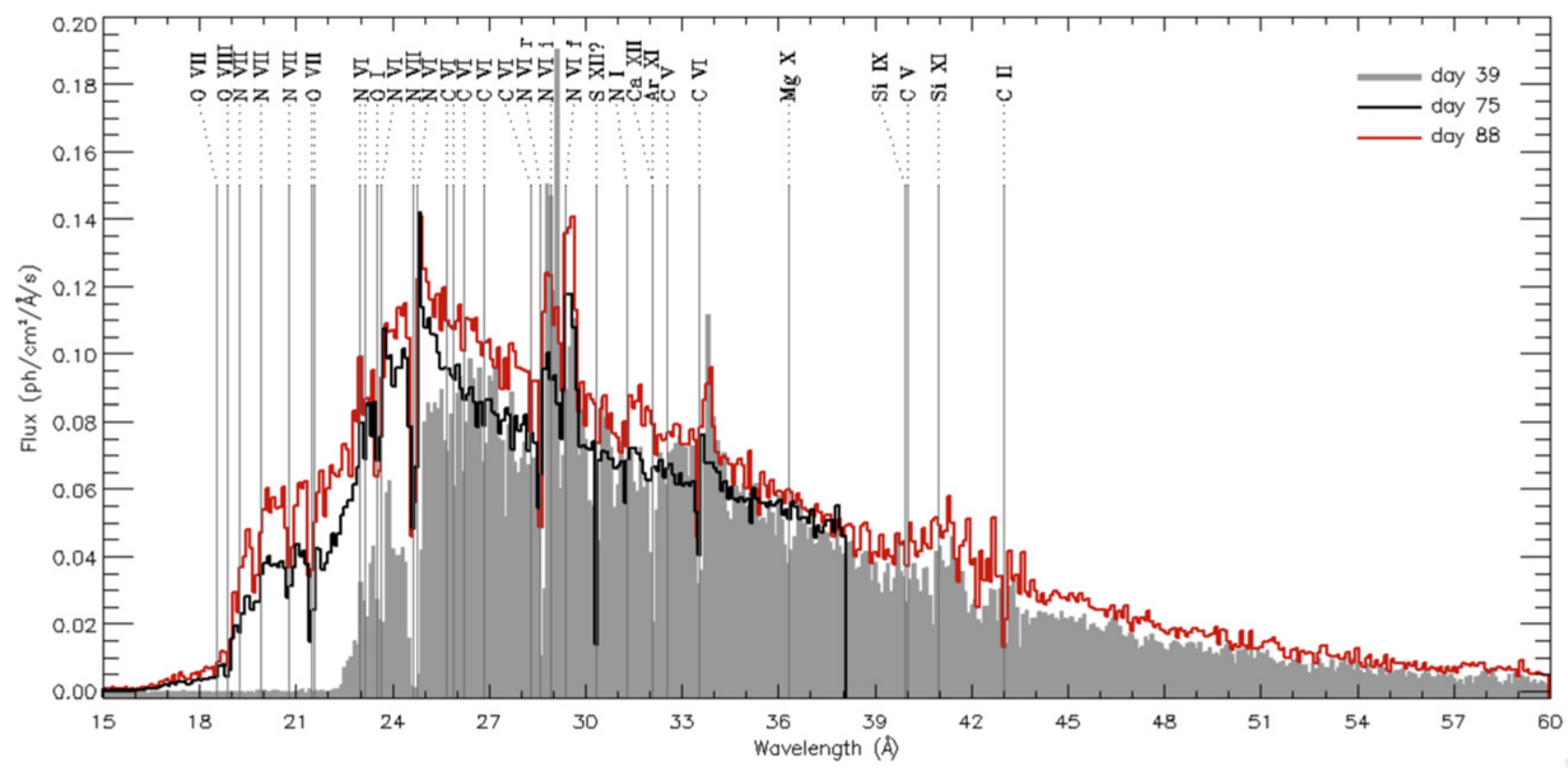

Figure 2. The flux-calibrated grating spectra of all three observations in units of counts of photons $(\mathrm{ph}) \mathrm{cm}^{-2} \mathrm{~s}^{-1} \AA^{-1}$. We marked the wavelengths of the main absorption lines we identify, assuming they are not at rest but blueshifted by $1800 \mathrm{~km} \mathrm{~s}^{-1}$, except for the lines of O I, N I, and C II that are indicated at rest wavelength, because we concluded that they are produced in the local interstellar medium.

these identifications, a custom-tailored atmospheric model with ad hoc, fine-tuned abundances will be crucial.

In addition to the blueshifted atmospheric lines, like in many other X-ray spectra of novae, we detect several absorption lines, at the rest wavelength, of transitions that occur with much lower ionization or excitation potentials, namely O I (23.508 $⿱$ ) and N I (31.28 $\AA$ ), and several features due to C II and C III around $42-43 \AA$. Below, we dedicate a separate section to the carbon features, because they have only been identified and measured very recently in the spectra of Galactic novae (Gatuzz et al. 2018). These spectral features and K-edges at low ionization are always observed at their rest wavelength and are thought to be produced in the interstellar medium (ISM) between the nova and us. They are not typical of novae; they are detected whenever there is a bright X-ray source, including many low-mass X-ray binaries and, more rarely, active galactic nuclei (AGNs), which act as lamps giving a strong backlight (see Gatuzz et al. 2015 and references therein).

A few features remain unidentified. These include several lines in the day 39 spectrum, which has the strongest absorption lines in the soft range: one at $25.93 \AA$ (accounting for blueshift, it corresponds to the unidentified $26.06 \AA$ feature in the list of Ness et al. 2011); two lines measured at 27.33 and $27.5 \AA$ that are close but not perfectly coincident, accounting for the blueshift, with unidentified lines of RS Oph (Ness et al. 2011); and finally, three lines at $30.87 \AA, 38.1 \AA$ and $39.2 \AA$.

\subsection{Comparison with Other Novae}

While the oxygen, nitrogen, and carbon absorption lines have been observed in most other novae, in Figure 4 we show that the SSS spectrum of day 39 appears extremely similar to the one observed at a later post-outburst epoch for KT Eri (day 84 post-outburst), as taken from the Chandra archive. Another nova X-ray spectrum that presents similarities is that of V4743 Sgr at day 180 (Rauch et al. 2010), but there was less flux at the longest wavelengths, and there were other differences in the observed features. The lines that we attributed to transitions of magnesium, argon, and, possibly, sulfur, are either missing or much less prominent in V4743 Sgr. Rauch et al. (2010) found solar sulfur and depleted magnesium with respect to solar values in V4743 Sgr. Sulfur, argon, calcium, and aluminum are intermediate atomic weight elements expected to be overabundant with respect to the solar value in oxygen-neon novae, but they should have solar abundance (or less) in novae on carbon-oxygen WDs (see, e.g., Starrfield et al. 2009). An accurate determination of the sulfur abundance will be particularly important because the ratio of its abundance over that of oxygen and aluminum is one of a few "nuclear thermometers" that constrain the degree of mixing of the accreted material with inner core elements (Kelly et al. 2013).

The intermediate-mass elements are synthesized on oxygenneon WDs, where neon-sodium and magnesium-aluminum cycles, which do not occur on carbon-oxygen WDs, operate in addition to the CNO cycle (José \& Hernanz 1998; Starrfield et al. 2009; Kelly et al. 2013). Magnesium is abundant in a superficial layer on oxygen-neon WDs, and as a consequence, atomic magnesium (the sum of the three isotopes ${ }^{24} \mathrm{Mg},{ }^{25} \mathrm{Mg}$, and ${ }^{26} \mathrm{Mg}$ ) is expected to be above solar abundance in oxygenneon novae (José \& Hernanz 1998; Kelly et al. 2013). Thus, we need to be able to model the spectrum sufficiently well to derive whether it is fitted with the range of abundances that would produce these lines, in order to be able to tell whether the underlying WD is oxygen-neon or carbon-oxygen. This "first-order" initial comparison indicates that V4743 Sgr, KT Eri, and N SMC 2016 at this stage in the evolution of each nova had approximately the same $T_{\text {eff: }}$ it was estimated to be $740,000 \pm 70,000 \mathrm{~K}$ in this spectrum of V4743 Sgr, and our preliminary result for $\mathrm{KT}$ Eri also indicates a $T_{\text {eff }}$ around 

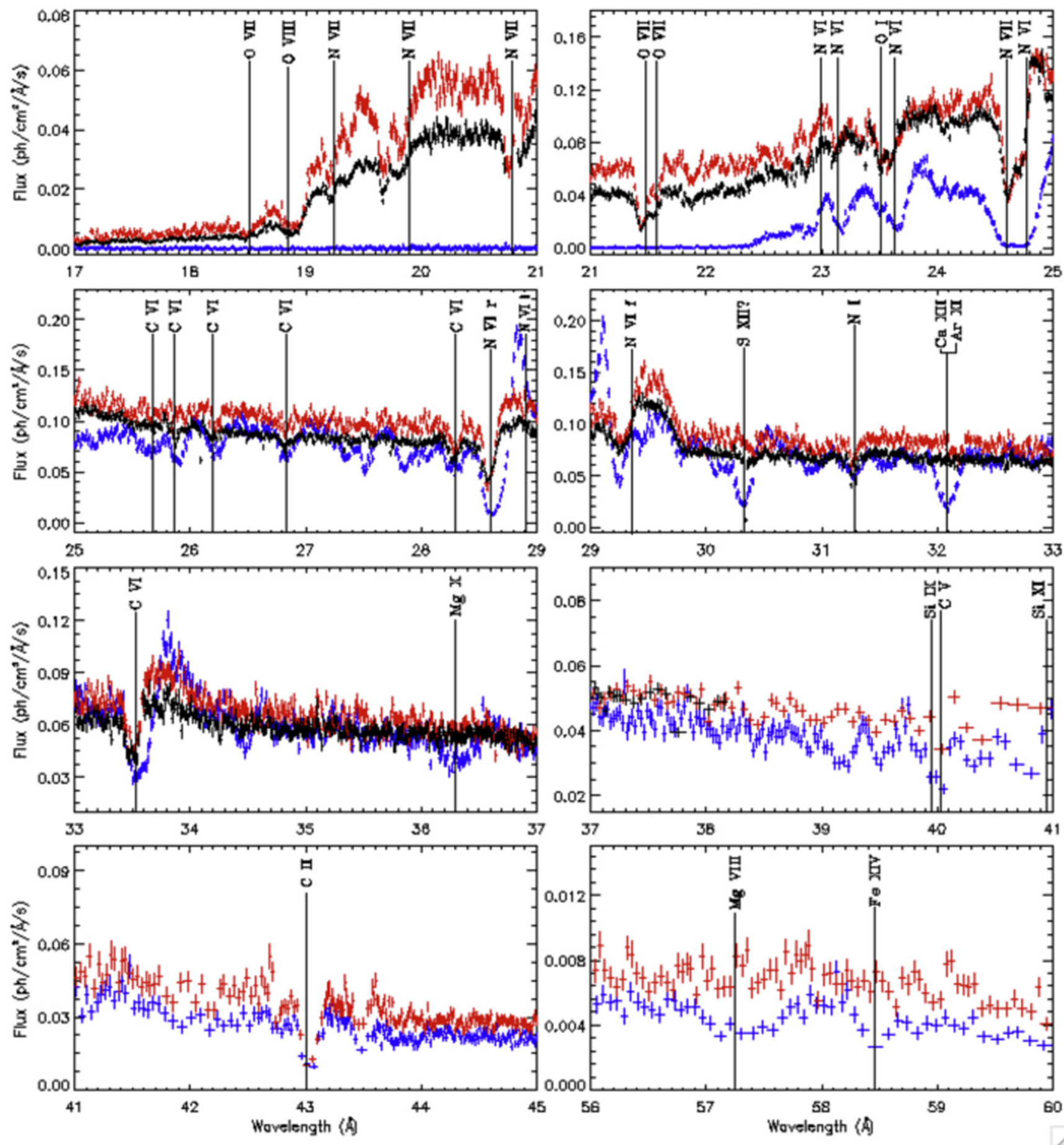

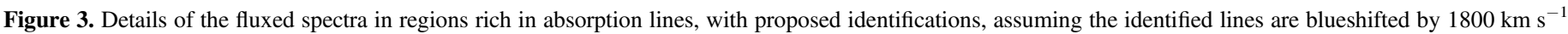

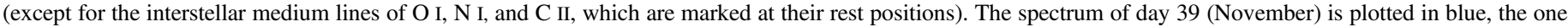

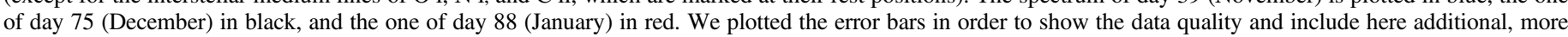

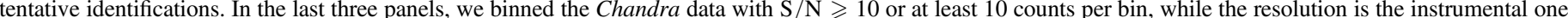
in the other panels for the Chandra data, and the XMM data were binned with at least $\mathrm{S} / \mathrm{N} \geqslant 7$ or 7 counts per bin.

$700,000 \mathrm{~K}$. However, $T_{\text {eff }}$, which at its maximum is an indication of the WD mass (Starrfield et al. 2012; Wolf et al. 2013), was still increasing to its peak in N SMC 2016. Both novae whose spectra we plotted in Figure 4, N SMC 2016 analyzed in this work and KT Eri, are likely to host oxygenneon novae. V4743 Sgr, instead, fitted by Rauch et al. (2010) with a model for a carbon-oxygen WD, with solar abundances of intermediate atomic mass elements and depleted magnesium, very likely occurred on a carbon-oxygen WD.

\subsection{Model Fitting}

In order to classify the nova correctly and to estimate the abundances in the hot atmosphere, including verifying the 


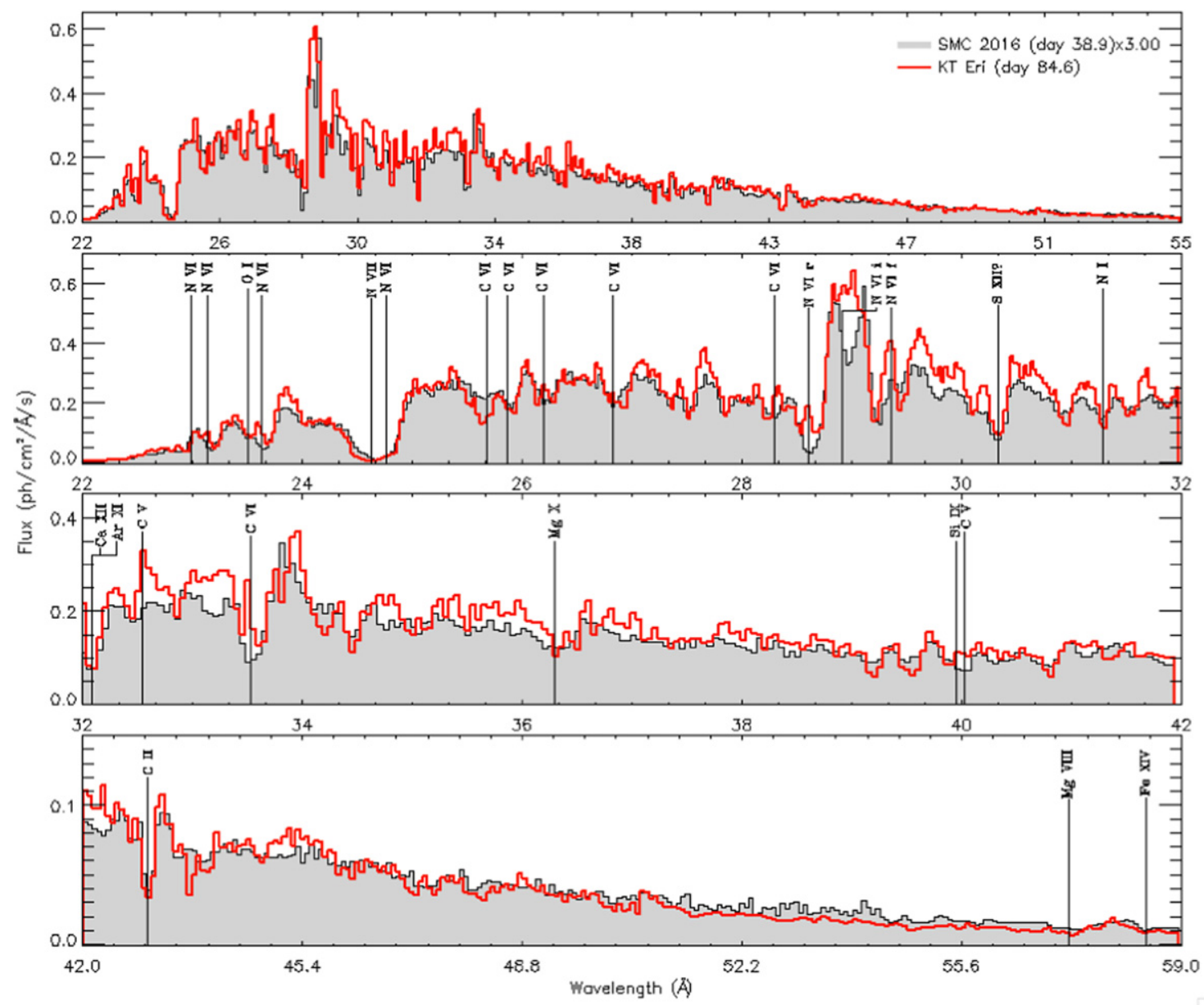

Figure 4. Comparison of the Chandra HRC-S+LETG spectra of N SMC 2016 at day 39 and KT Eri at day 84 . Several absorption lines (assumed to be blueshifted by $1800 \mathrm{~km} \mathrm{~s}^{-1}$ ) are also indicated, together with the rest-wavelength positions of the ISM lines identified above.

possible oxygen-neon WD nature, a physical model is necessary. For this article, we experimented with two different models: the non-local thermodynamical equilibrium model atmosphere TMAP (Rauch et al. 2010) and the synthetic models for expanding atmospheres, the "wind-type" (WT) model of van Rossum (2012). Since it takes considerable effort to produce new model grids with different sets of abundances, we used the grids already available in the above papers in order to understand whether these models are at all viable for this nova. A detailed abundance study requires extending the grids of models to find the parameters that reproduce our spectra; additional work is in progress and will be the subject of a forthcoming paper (T. Rauch et al. 2018, in preparation).

A basic question we would like to answer is: given the blueshift of the absorption features, what is the correct way to model this WD atmosphere from which an outflow is still present? The radiation-driven wind models (see, e.g., Hachisu et al. 2007) predict that the end of mass loss occurs around the time the WD appears as an SSS, but in the SSS, we still find blueshifted absorption lines in this and in other novae. A typical case is RS Oph, which was analyzed by Hachisu et al. (2007), who inferred the end of mass loss, while the X-ray spectra measured by Ness et al. (2007) and Nelson et al. (2008) still showed blueshifted absorption features. Although the pseudo-photosphere of the nova has clearly contracted, as foreseen by all models, and the WD radius has shrunk from that of a red giant configuration at the time of the optical luminosity peak, becoming very compact, as predicted by the models for the constant bolometric luminosity phase (e.g., Starrfield et al. 2012; Wolf et al. 2013), the blueshifted absorption lines can be explained if there is still an outflow from an expanding atmosphere. This residual, late-phase outflow has not been predicted by any models, but the blueshift of the absorption features has indeed been detected in most SSS high-resolution spectra of novae (see, among others, Rauch et al. 2010; Ness et al. 2011; Orio 2012; Orio et al. 2013). It is interesting to note that the lines are often blueshifted with about the same velocity observed in the emission lines of the optical spectra of the outburst.

When the WD photosphere has shrunk back to an almost "normal" WD radius, the amount of mass lost in the wind is likely to be very small. With a large mass outflow, the absorption features would not be so deep, and the profile would not remain similar to the static atmosphere case. Therefore, we reason that a first attempt at a fit can be done with a static atmosphere. Most X-ray grating spectra of novae are in fact very similar to CAL 83, a steadily burning source that does not present mass loss, albeit generally the novae we have observed are at higher $T_{\text {eff }}$ than CAL 83, which was found to have $T_{\text {eff }} \simeq 550,000 \mathrm{~K}$ (Lanz et al. 2005). The TMAP code nova model grid was initially developed with the aim of reproducing the abundances of V4743 Sgr (Rauch et al. 2010); the published grid includes various combinations of highly nonsolar abundances as calculated for the burning layer in models 


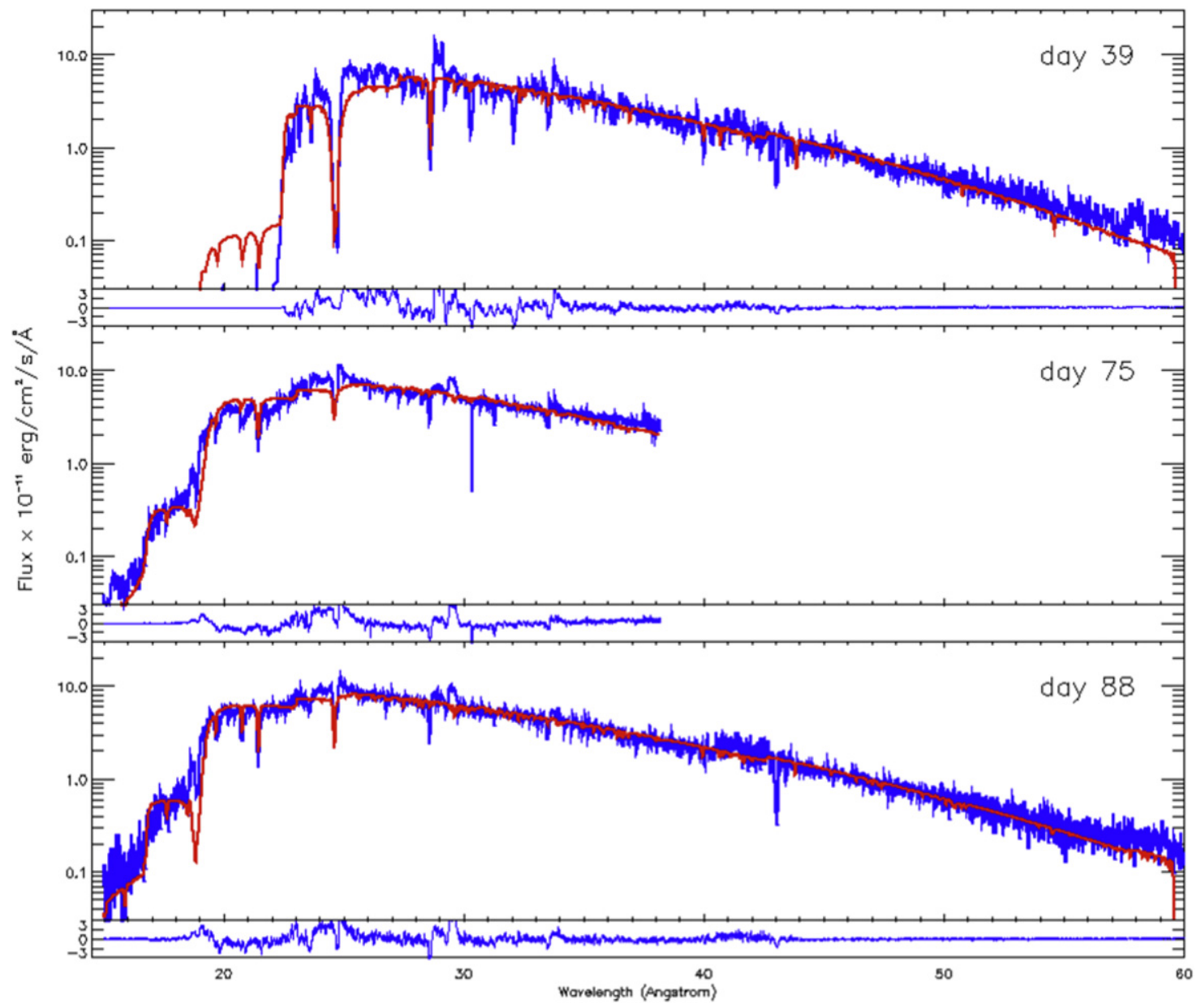

Figure 5. In the top panels, the observed spectra of N SMC 2016 taken on days 39 and 88 with the Chandra HRC-S+LETG setup and on day 75 with the RGS of $X M M$-Newton are traced in blue for each date. The red lines show the XSPEC best fit with a TMAP model with $\log (g)=9$ and the parameters in Table 2 . The fit has been obtained by artificially moving the atmospheric absorption lines with respect to the original model in order to match the observed blueshift. For each spectrum, the lower panel shows, in linear scale, the residuals, namely the difference between the data and the model.

of carbon-oxygen WDs by Prialnik and coauthors (obtained directly from them). The other grid of models we used was obtained with the WT expanding atmosphere code, which includes a larger number of atomic species but has only solar abundances (van Rossum 2012).

In order to take the intervening column density into account, we used the Tübingen-Boulder absorption model and performed the calculation with the related routines TBNEW in IDL and TBABS in XSPEC (see Wilms et al. 2000). In Figure 5, we show the TMAP fits for all observations, whose parameters are given in detail in Table 2, and in Figure 6, we present the WT fits. The Chandra spectra, providing a softer range, allow us to evaluate the absorbing column density of the equivalent neutral hydrogen $N(\mathrm{H})$ better than the XMM-Newton one. We note that Aydi et al. (2018), referring also to Haschke et al. (2012), adopted a value $A_{V}=0.11 \pm 0.06$, corresponding to a very low column density, $N(\mathrm{H})=1.7 \times 10^{20} \mathrm{~cm}^{-2}$ (following Predehl \& Schmitt 1995). Because in X-rays we are observing deeply inside the shell, there may be additional column density, but it has to be low enough for the material to be optically thin to supersoft X-rays. Despite the unexplained velocity of the absorption lines, we obtained a much better fit to the observed spectra with TMAP (with the caveat of artificially shifting the absorption features to match the data; see Figure 5) than with the WT models (see Figure 6).
Table 2

Parameters of the Best Fit Obtained for N SMC 2016 with TMAP

\begin{tabular}{lccccc}
\hline \hline Day & $T_{\text {eff }}(\mathrm{K})$ & $\begin{array}{c}N(\mathrm{H}) \\
\left(10^{20} \mathrm{~cm}^{-2}\right)\end{array}$ & $\begin{array}{c}F(\text { unabs) } \\
\left(\mathrm{erg} \mathrm{s}^{-1}\right)\end{array}$ & {$\left[\mathrm{N} / \mathrm{N}_{\odot}\right]$} & {$\left[\mathrm{C} / \mathrm{C}_{\odot}\right]$} \\
\hline 39 & 716,000 & 4.92 & $2.16 \times 10^{-9}$ & 1.803 & -1.513 \\
75 & 852,000 & 5.44 & $2.72 \times 10^{-9}$ & 0.937 & -0.529 \\
88 & 904,000 & 4.04 & $2.59 \times 10^{-9}$ & 1.159 & -0.596 \\
\hline
\end{tabular}

Note. The unabsorbed flux is calculated in the $12-124 \AA$ range $(\approx 0.1-1 \mathrm{keV})$ and represents more than $95 \%$ of the bolometric flux.

The TMAP fit was done in XSPEC. The best fits we obtained, tested according to two methods of analysis, the $\chi^{2}$ and the Cash statistics (Cash 1979), are shown in Table 2. These fits yield a temperature of $716,000 \mathrm{~K}$ for day 39 , $852,000 \mathrm{~K}$ for day 75 , and $904,000 \mathrm{~K}$ for the last observation of day 88 done with Chandra. The fits are not perfect, but they approximate the shape of the continuum and the strongest absorption features quite well. However, the fits yield excess flux both on the soft portion of the spectrum for the spectra of days 75 and 88, and also excess flux on the "hard" side for day 39. For day 39, if we decrease the temperature from 716,000 to $650,000 \mathrm{~K}$, the N VII K-edge that is responsible for cutting the hard X-ray flux shortwards of $18.59 \AA$ is matched, but at the 


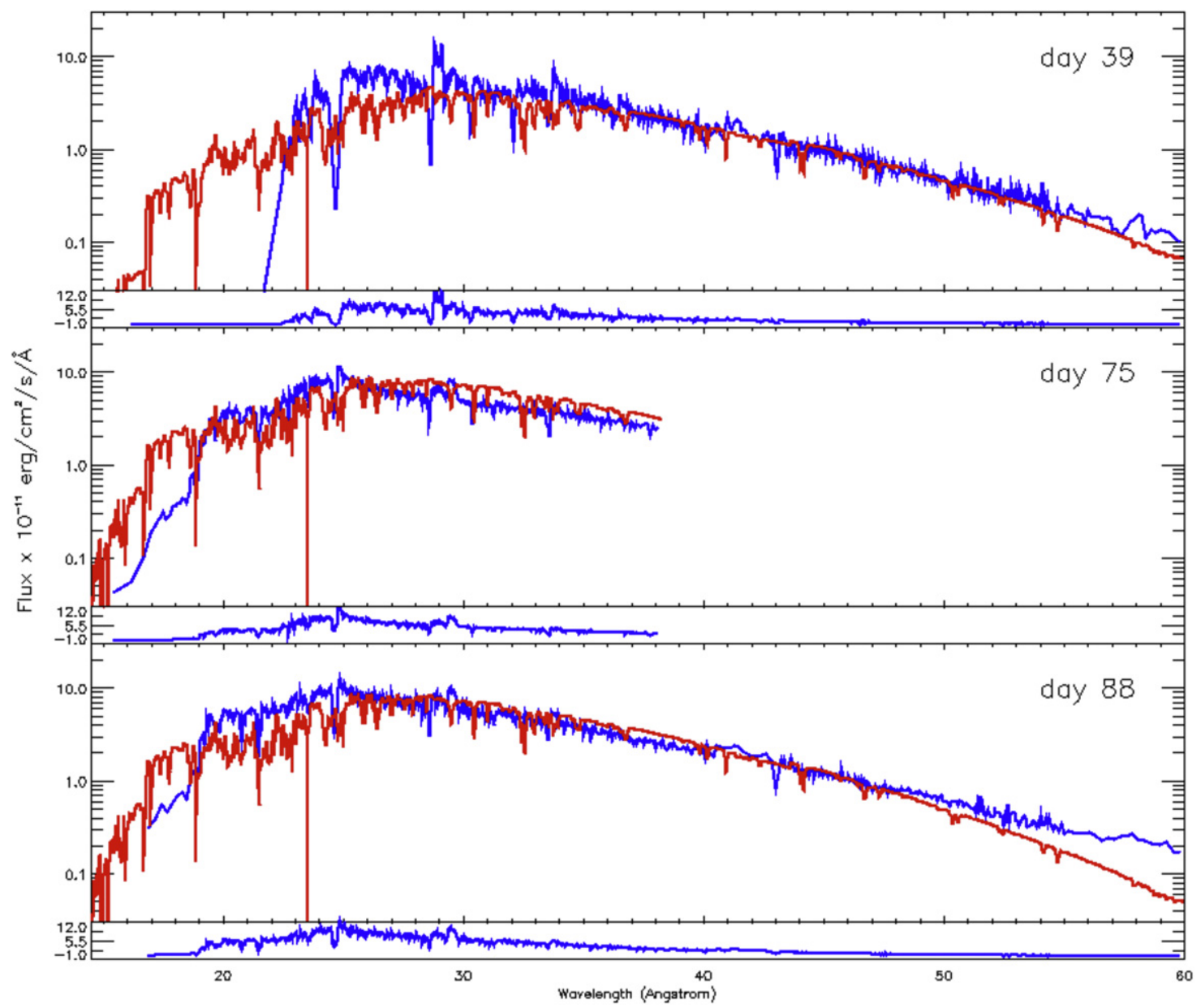

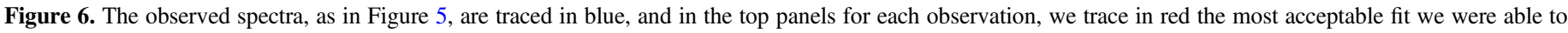

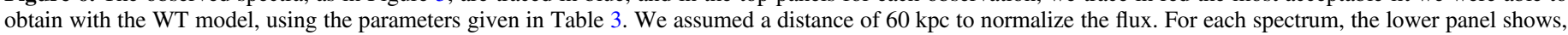
in linear scale, the residuals, namely the difference between the data and the model.

expense of underpredicting even more the soft flux longwards of $45 \AA$. Decreasing the value of the absorbing column $N(\mathrm{H})$ does not lead to a perfect fit in this soft part of the spectrum. The main problem here is that the grid step of $50,000 \mathrm{~K}$ results in too large an uncertainty in $T_{\text {eff }}$ to have sufficient precision to match the absorption edges perfectly. The Swift XRT trend suggests that during the day 75 observation, the temperature may have still been lower than on day 88 , but because of the large step in $T_{\text {eff }}$ in the grid, we can only constrain it to be in the 850,000-900,000 $\mathrm{K}$ range for both days. We note that the model misses a feature of He-like nitrogen at about $28.78 \AA$ that is still observed on days 75 and 88. This may mean that we have overestimated the temperature, due to a lack of a suitable grid step, or that the best fit underestimates the nitrogen abundances. For a peak SSS temperature in the ballpark of the value we obtained, the WD mass is $1.2 \leqslant m(\mathrm{WD}) \leqslant 1.3 M_{\odot}$ following Wolf et al. (2013) and $1.25 M_{\odot}$ following Starrfield et al. (2012), consistent with the values inferred by Aydi et al. (2018).

In the publicly available grid of models, the parameters that mainly vary are the carbon and nitrogen abundance. While on day 39 the best fit was obtained with the highest ratio of $\mathrm{N} / \mathrm{C}$ (in mass relative to the solar value), the ratio was lower on days 75 and 88, and this may imply that the atmospheric layer was mixing with newly accreted material from the companion. At a distance of $60 \mathrm{kpc}$, the unabsorbed flux on day 38 implies an
X-ray luminosity of $9.26 \times 10^{38} \mathrm{erg} \mathrm{s}^{-1}$ in the $12-124 \AA$ range (equal to almost all the bolometric luminosity) and of $1.11 \times 10^{39} \mathrm{erg} \mathrm{s}^{-1}$ on days 75 and 88 . The fits indicate only a modest increase in unabsorbed flux from day 39 to day 75 , consistent with the WD photospheric radius still shrinking at approximately constant bolometric luminosity. We notice that these values are above Eddington level and indicate a larger radius than that of a WD of $1.25 M_{\odot}$. After the 40 days of super-Eddington luminosity inferred in the optical and UV range, the nova luminosity exceeded the Eddington limit at least until day 88 after having shifted the peak of emission to the X-rays; because the Swift XRT light curve (Figure 1 and Aydi et al. 2018) implies that the SSS did not decline and remained close to the level of day 88 , the luminosity must have been above the Eddington level even over five months after the outburst. This implies that the stellar configuration was not yet stationary and is consistent with the outflow indicated by the blueshifted lines.

It is interesting to note that the absorption features of the nova, although blueshifted, are as deep as predicted by the static atmosphere model. In the day 39 spectrum, the N VII $24.78 \AA$ line even appears saturated. A likely explanation is that little mass is flowing out, because only in such a case does the WT model become more similar to a static one, and this would be the reason why the static model approximates the observed spectrum reasonably well. 
Table 3

Parameters of the Most Acceptable Fits We Obtained for N SMC 2016 with the WT Model

\begin{tabular}{lccccc}
\hline \hline Day & $\begin{array}{c}N(\mathrm{H}) \\
\left(10^{20} \mathrm{~cm}^{-2}\right)\end{array}$ & $v_{\mathrm{ej}}\left(\mathrm{km} \mathrm{s}^{-1}\right.$ & $\log (g)$ & $\dot{m}\left(M_{\odot} \mathrm{yr}^{-1}\right)$ \\
\hline 39 & 650,000 & 5.00 & 1800 & 8.9 & $7.6 \times 10^{-9}$ \\
75 & 700,000 & 6.00 & 1800 & 9.01 & $7.6 \times 10^{-9}$ \\
88 & 700,000 & 6.00 & 1800 & 9.01 & $7.6 \times 10^{-9}$ \\
\hline
\end{tabular}

Note. For days 75 and 88, the temperature is the highest available in the grid, because no model was calculated with a higher $T_{\text {eff }}$. We assumed a $60 \mathrm{kpc}$ distance to the nova.

Because the WT model is not available in the standard HEASOFT XSPEC software or in the other spectral packages, we calculated the convolution of the flux at each wavelength with the absorption (or transmission) function by using IDL. In a static model, the effective gravity is dependent on the temperature $T_{\text {eff, }}$, but in the WT model, there are more parameters (the velocity and the mass outflow rate $\dot{m}$ ), although the parameters are not independent from each other. For a given temperature and effective gravity, the mass outflow rate is determined by the velocity of the wind, but in the WT model, the wind velocity does not correspond to the observed blueshift of the absorption features, because the lines are produced in an extended, outflowing medium with a complex structure. Since the model is not included in a spectral fitting package, we first fitted the data with different sets of parameters in the grid, then compared them by eye. The fits we found most acceptable are plotted in Figure 6. There is a sufficient difference between certain sets of models that we could rule out a large number of them, namely those with a high mass outflow rate and low effective gravity. Thus, we focused on fits with the "dlg-03" and "dMv442" series, which include the combination of the highest effective gravity and the lower mass outflow rate for a given velocity. Because the models predict the absolute flux and we know the distance to the source, we found that the temperature that is necessary to match the observed flux produces too hard a spectrum. The particular structure of the medium in which the transitions occur in the WT models smears the absorption edges and cannot reproduce them. An "experiment" with an XSPEC blackbody model with overlapping absorption edges showed us that the latter are very sharp even with solar abundances; therefore, we suggest that it is the wind structure that cancels, or rather "velocity-smears," the absorption edges in the WT model, not the lack of enhanced abundances. Van Rossum (2012) found that he could fit one of the V4743 Sgr spectra with a systematically lower temperature than the static atmospheric models, but in this case, we cannot lower $T_{\text {eff }}$ or else the flux would be so low that the nova should be in the Galaxy (this has been was ruled out by Aydi et al. 2018). Thus, in practice, the high temperature is constrained by the absolute luminosity. For instance, for the first spectrum, a fit with $T_{\text {eff }}=450,000 \mathrm{~K}$ would imply a total absorbed luminosity that is lower than observed by two orders of magnitude at a distance of $60 \mathrm{kpc}$. The nova would have to be Galactic, but even with this assumption, we still do not obtain any match with the observed spectral features. We note that, even if we match the flux to fit a distance of only $45 \mathrm{kpc}$, which was not completely ruled out by Aydi et al. (2018), the $T_{\text {eff }}$ indicated by the model for the first observation would not be lower than $650,000 \mathrm{~K}$. The highest temperature in the grid is $750,000 \mathrm{~K}$, and in the middle and lower panels of Figure 6, we show how it fits the second and third observations (poorly; however, clearly it is only a lower limit). The parameters of the WT fits are reported in Table 3.

The WT models also fail to reproduce the observed blueshift and depth of the lines. In fact, as the surface gravity decreases and the density structure changes in the wind with respect to a static atmosphere, the absorption features tend to become much shallower and are at times accompanied by emission wings in P-Cyg profiles, causing only a modest blueshift of the absorption features that does not equal the wind velocity.

There are structures in the observed spectra that appear to consist of emission lines, at least around $29 \AA$, between 33 and $34 \AA$, and possibly also a little redwards of about $43 \AA$, and that no models can reproduce (we note, however, that the LETG spectrum is noisy around $43 \AA$ because of the very low effective area, due to instrumental (HRC-S) absorption by the carbon K-edge at $43.6 \AA$ ). A combination of static atmosphere and plasma in collisional ionization equilibrium in the ejecta has been found to be present in several novae: U Sco (Orio et al. 2013), T Pyx (Tofflemire et al. 2013), and V959 Mon (Peretz et al. 2016), but some of the apparent emission lines in N SMC 2016 are in the range below $40 \AA$ and are not reproduced with the plasma temperature of the available "packaged" data analysis models of collisional ionization equilibrium.

Our proposed future course of action to reveal the chemical composition of this nova WD is to first obtain a static model that fits the continuum and the absorption features better than the existing ones: this is currently being tried with new sets of parameters in the TMAP model (T. Rauch et al. 2018, in preparation). Only after this is achieved will we will try to analyze whether photoionization or collisional ionization can explain the residual emission lines, and whether they may originate in the ejecta, far from the WD, or instead are connected with the residual wind from the WD.

\subsection{Lines Originating in the ISM}

In Figures 2-4, we marked the lines of O I and N I at rest wavelength, because these are known to be typically produced in the ISM and to appear in the X-ray grating spectra of many different X-ray sources, both Galactic (usually low-mass X-ray binaries; see Gatuzz et al. 2016; Gatuzz \& Churazov 2018) and extragalactic (AGNs; see, e.g., Nicastro et al. 2016a, 2016b; Gatuzz \& Churazov 2018). For these reasons, and also because these lines are produced with a photoionizing source of much lower temperature than needed for all other lines in the spectrum, we rule out that they originate in the nova and are intrinsic to it.

Thanks also to recent updates in the calibration package CALDB that better account for the HRC $43.6 \AA \mathrm{C}$ I absorption edge, making the calibration at the nearby wavelengths more secure, we were also able to identify C II and C III features in the 38-44 $\AA$ range which have been recently detected and measured in the ISM by Gatuzz et al. (2018) using Galactic novae as lamps. These authors (and several others quoted in their paper) did not find evidence of C I features in the ISM, implying that neutral carbon has a very low column density, lower than $10^{14} \mathrm{~cm}^{-2}$ along multiple lines of sight. However, Gatuzz et al. (2018) were able to detect several features of C II and C III by using the Chandra HRC+LETG spectra of four 

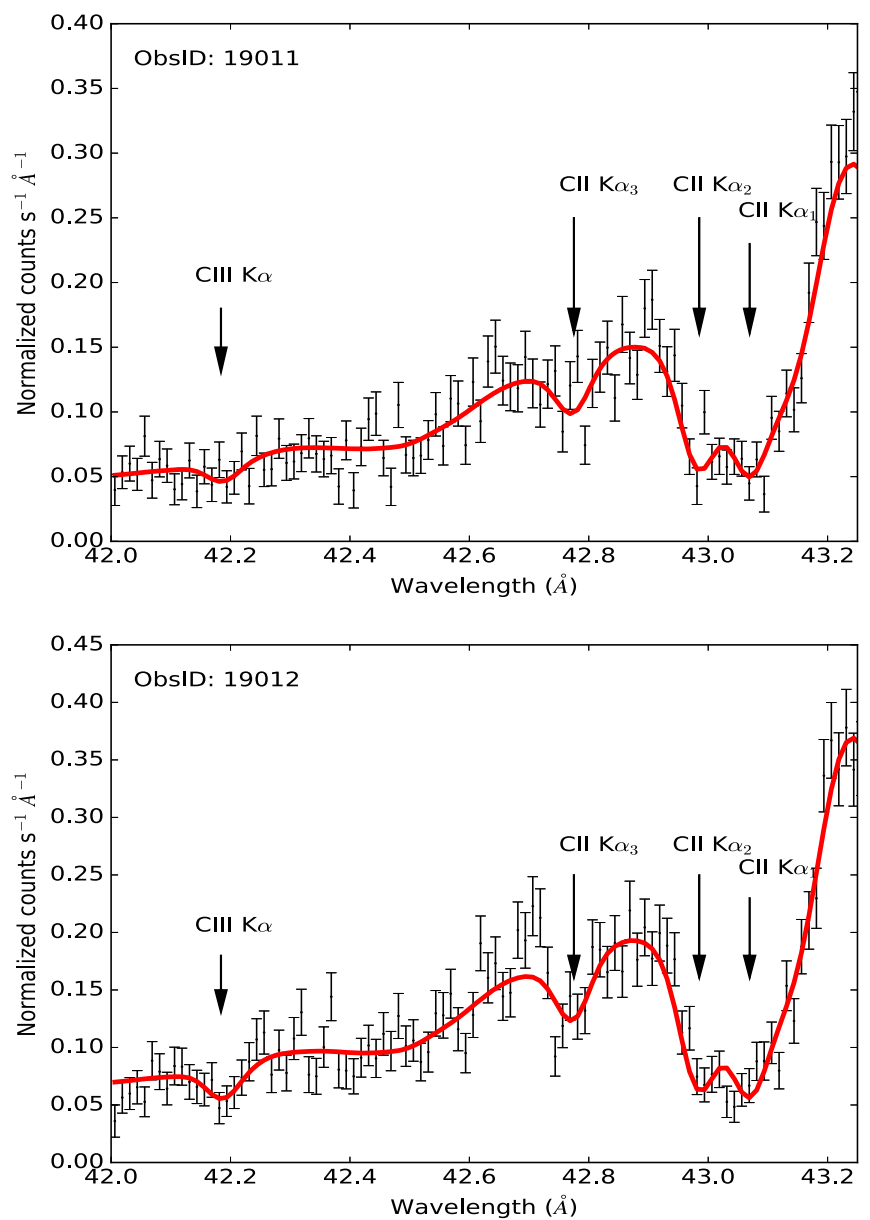

Figure 7. The red line shows the best-fit results for the C II K-edge wavelength region, including the atomic data benchmarking correction (Gatuzz et al. 2018). The top panel presents (in black) the day 39 data; the lower panel shows the data of day 88 .

Galactic novae and the ISMabs model (Gatuzz et al. 2015). The spectrum of Nova SMC 2016 offers the possibility of calibrating the column density of these ions along a new line of sight, away from the Galactic center. We identified the $\mathrm{C}$ II and $\mathrm{C}$ III $\mathrm{K} \alpha$ triplets as well as the $\mathrm{C}$ II and $\mathrm{C}$ III $\mathrm{K} \beta$ resonances. The high resolution achieved by the Chandra HRC-S+LETG instrument allows a detailed analysis of such features. We have not identified $\mathrm{CI}$ absorption lines in the spectra of $\mathrm{N} \mathrm{SMC}$ 2016. In Figure 7, we zoom into this specific spectral region for a clear understanding of these features, and Table 4 lists the column densities obtained for each observation. Additional future observations of X-ray-luminous novae located in the Magellanic Clouds, at different $T_{\text {eff }}$, will be very useful for the estimation of the ISM chemical composition in these lines of sight (e.g., ionization fractions, abundances, and column densities).

\section{Timing Analysis}

Figure 8 shows the light curves measured in the three observations. The Chandra light curves are the zero-order light curves measured with the HRC-S camera. The XMM-Newton light curve is the RGS first-order one, which, despite a time resolution of only $5 \mathrm{~s}$, is not affected by pile-up like the EPIC light curves. We restricted our analysis to the gratings' observations. There are large irregular fluctuations: the count
Table 4

ISM Carbon Column Density Best-fit Results. The Units are $10^{16} \mathrm{~cm}^{-2}$

\begin{tabular}{lcc}
\hline \hline Day & $N(\mathrm{C}$ II $)$ & $N(\mathrm{C}$ III $)$ \\
\hline 38 & $8.75_{-1.44}^{+1.28}$ & $0.29_{-0.26}^{+0.45}$ \\
88 & $10.28_{-1.22}^{+1.35}$ & $0.59_{-0.34}^{+0.50}$ \\
\hline
\end{tabular}

rate varies by factors of 3 on day 39, by 1.5 on day 75 , and by almost 2 on day 88. We also extracted both Chandra light curves of days 39 and 88 between 15 and $33 \AA$ and compared them with the light curves between 33 and $60 \AA$. We also compared the light curves of day 39 split differently, in the $20-32 \AA$ range and in the $32-50 \AA$ range, but we did not find that the modulations were larger, with stronger dimming, in the softer bands, as expected if the fluctuations are caused by variable absorption. We repeated the experiment with the $X M M$ light curves of day 75 , comparing the 15-27 $\AA$ wavelength range light curve with the $27-38 \AA$ light curve, but even in this case there was no indication of a larger fluctuation in the lower energy band. We also extracted two separate spectra for day 75 , one for count rates above 50 counts s$^{-1}$ and one for count rates below this level, and measured no significant difference in the spectra except for the continuum flux level.

The variability appears to occur on different timescales, but we could not find an obvious coherent periodicity. We performed a periodogram calculation with the Lomb-Scargle algorithm (Scargle 1982), because it is suitable for data that are not equally spaced in time. The XMM-Newton has a high background or other "flagged" intervals that could not be used, causing gaps in the light curve, so this algorithm is well suited.

For a more detailed timing analysis, we used power density spectra (PDS) instead of standard periodograms. This approach is suitable for studying quasiperiodic oscillations (QPOs) or red noise. We estimated the PDS as in Dobrotka et al. (2017), i.e., we split the light curve into $n_{\text {div }}$ subsamples (subdivision in time ranges), then we calculated periodograms in log-log space for every subsample, averaged them, and finally binned the data in equally spaced bins if a minimum number of points per bin is fulfilled (otherwise, the bin is larger). Usually, the lower frequency end and the frequency resolution of the periodogram are determined by the length of the exposure and the highfrequency end by the Nyquist frequency. For our analysis, instead of the latter, we chose the frequency at which the periodogram trend appears to become almost constant, indicating pure white noise. The standard periodograms do not show any significant peaks, suggesting that there is no coherent periodicity.

Figure 9 shows the PDS calculated using all three light curves, with $n_{\mathrm{div}}=1-3$. Note that the light curve of $X M M$ Newton is binned every $10 \mathrm{~s}$, the first Chandra light curve every $16 \mathrm{~s}$, and the second Chandra light curve every $50 \mathrm{~s}$. The trend is linear, suggesting that in all three cases we are only measuring red noise. This trend is shown by fitted broken power-law fits ${ }^{22}$ (red lines).

However, some trend deviation or power excess is noticeable in two cases. For day 39, we fitted these PDSs with a broken power law with an additional Lorentzian (the blue line in Figure 9). This additional component suggests the presence of a QPO, and the fits show marginally improved $\chi_{\text {red }}^{2}$ values. The

\footnotetext{
22 Two linear functions with different slopes before and after break frequency, in $\log -\log$ space.
} 


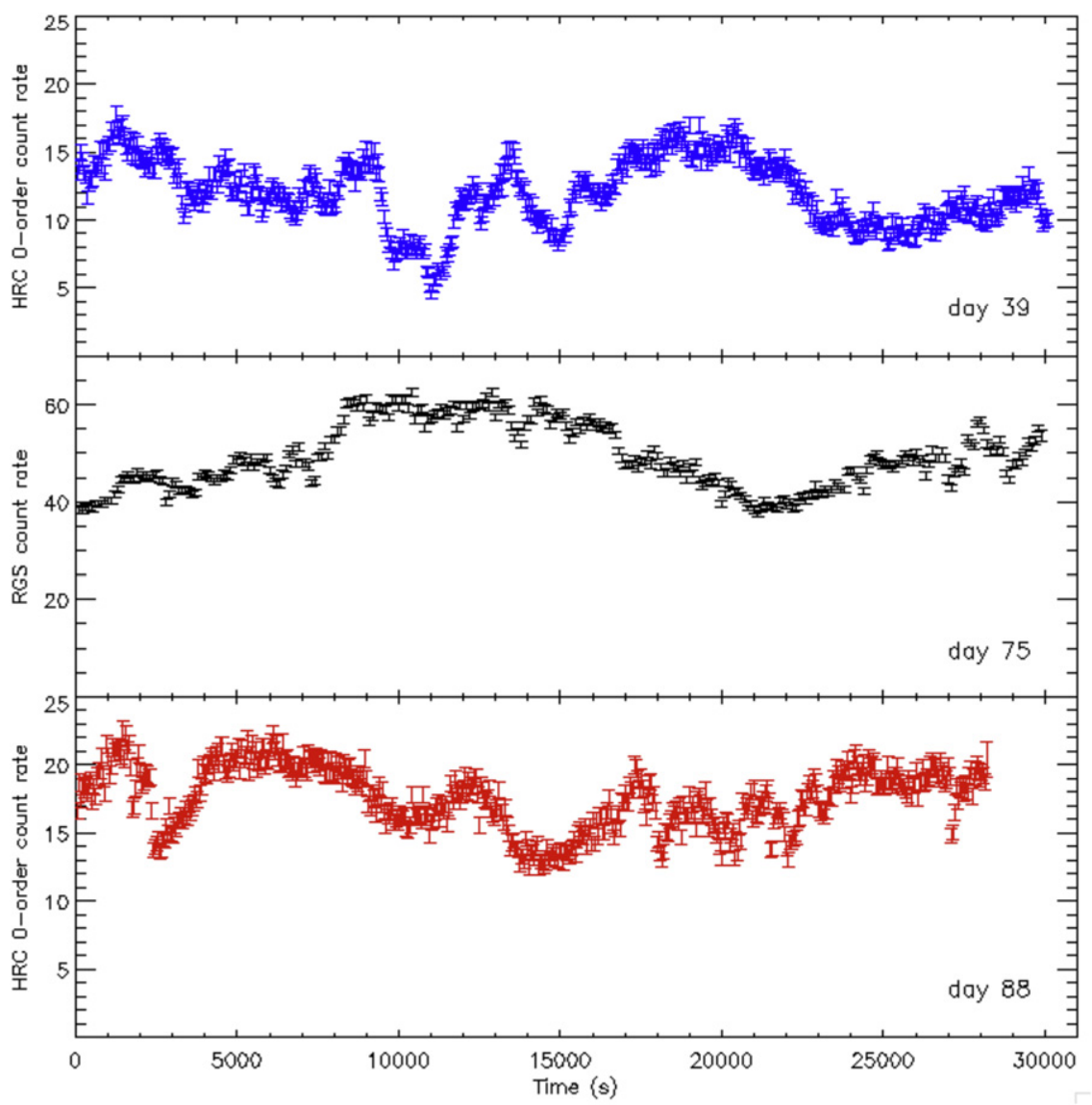

Figure 8. Light curves measured with the Chandra HRC-S camera as "zero order" in $15-80 \AA$ on days 39 and 88 , and with the XMM-Newton RGS $(+1$ and -1 orders, added) on day 75 . For plotting purposes, we have binned the HRC data every $50 \mathrm{~s}$, and the RGS data every $100 \mathrm{~s}$ (we used different binnings in the data analysis; see text).
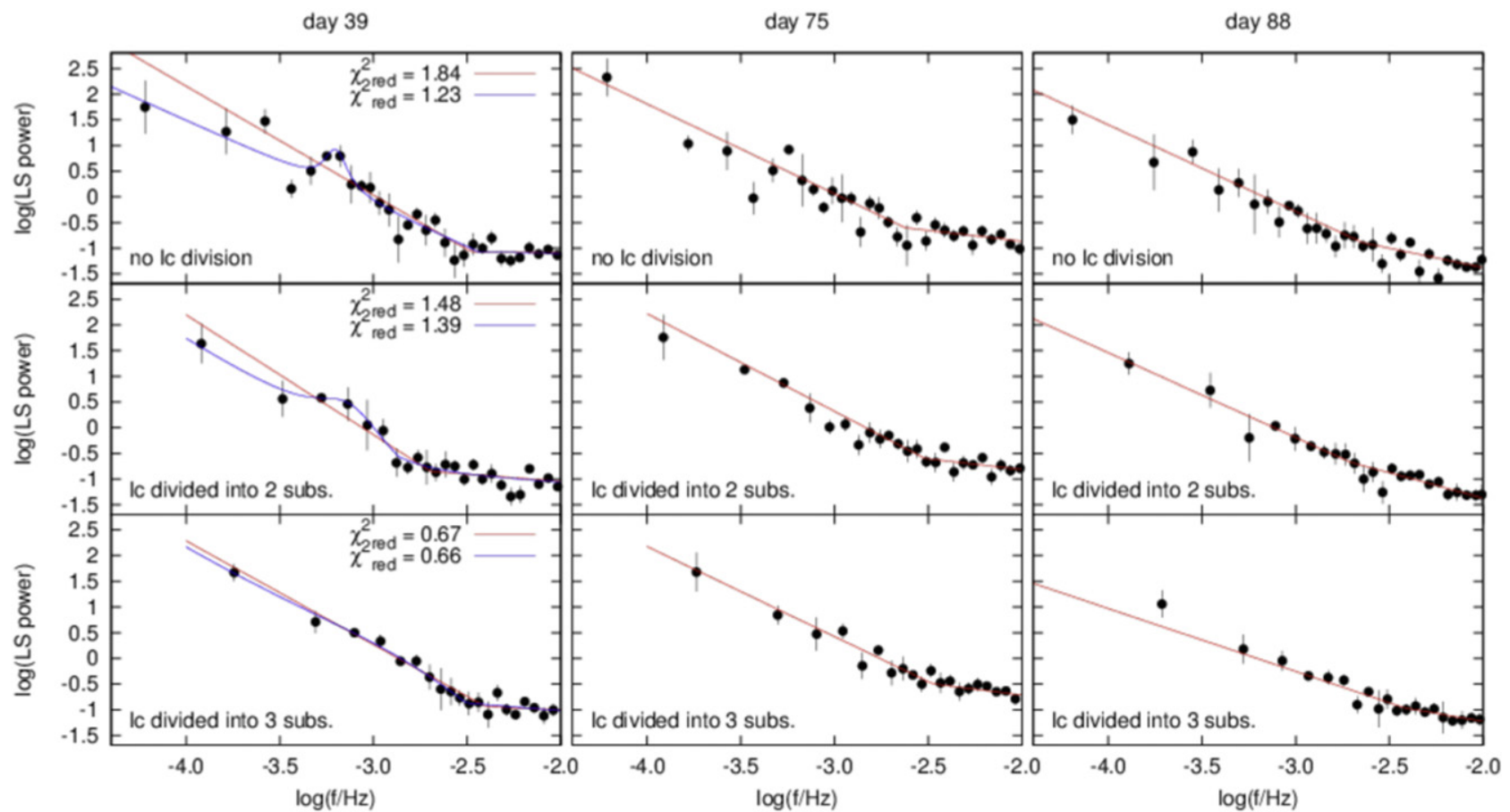

Figure 9. For all three light curves, we show here the PDS estimates obtained with different intervals in which the the light curves were split. The data points are the averaged values per frequency bin; the error of the mean is our uncertainty estimate. The red lines show a broken power-law fit and evidence the red noise below $\log (f / \mathrm{Hz})=-2.5$. The blue line in the panels on the left shows the same broken power-law fit with an additional Lorentzian, used to describe a possible power excess below $\log (f / \mathrm{Hz})=-3.0$ on day 39 , illustrating the effect of the light-curve splitting procedure. 


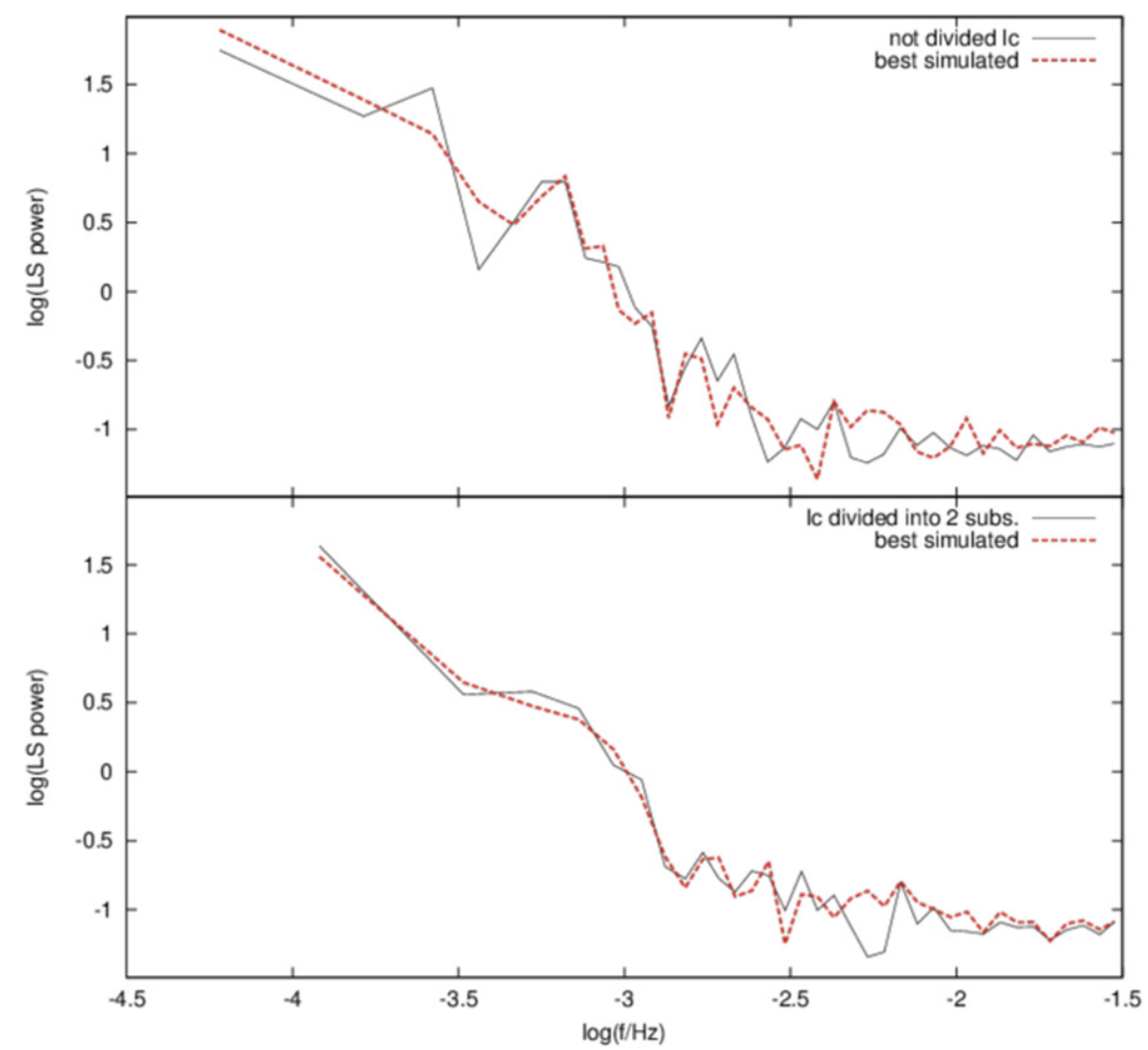

Figure 10. Observed vs. simulated PDSs (best cases chosen among 10,000 simulations), referring to the upper and middle panels in the middle column of Figure 9 , only for day 39.

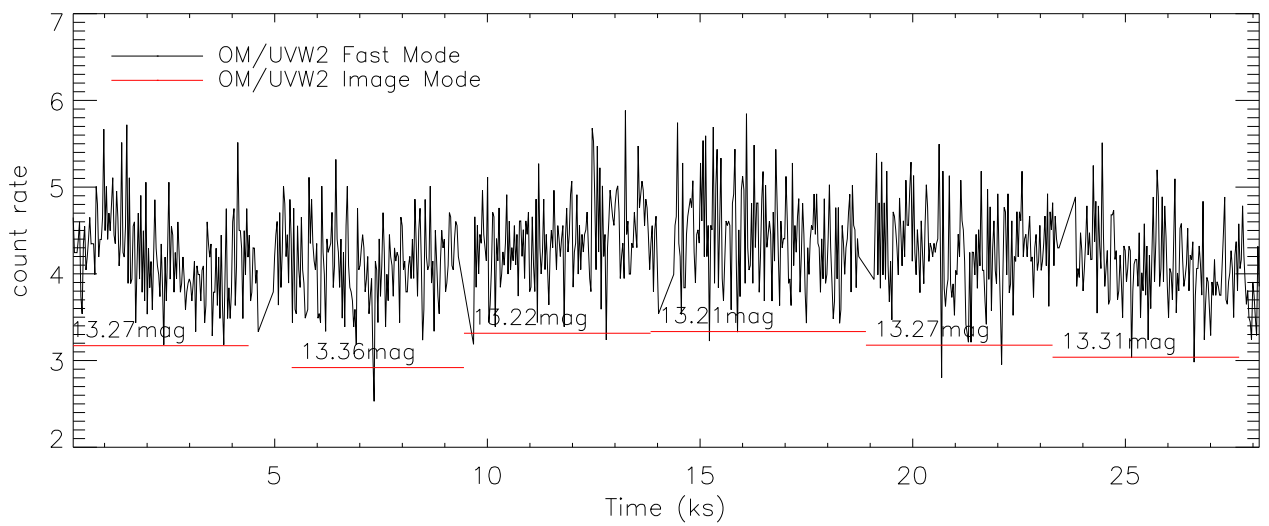

Figure 11. The $U V W 2$ filter light curve observed with the XMM-Newton OM on day 75 in fast mode and the magnitudes derived in the given intervals in imaging mode, as described in the text.

improvement decreases with increasing $n_{\text {div }}$. Is this power fluctuation real, or is it the result of a random process? In order to answer this question, we performed 10,000 simulations of the light curves using both a simple broken power law and the method of Timmer \& Koenig (1995). The best result we obtained in simulating the PDS in two cases, with the day 38 Chandra data, is shown in Figure 10. The power fluctuations can be explained with a random process, and we conclude that the variability in all three observations is dominated by a red noise.

Finally, we also calculated the PDS of the optical light curve obtained with the XMM-Newton OM. The light curve is presented in Figure 11. We detected only red noise for frequencies lower than $0.001 \mathrm{~Hz}$. Higher frequencies are dominated by Poissonian noise.

\section{Discussion and Conclusions}

Using spectral fits to the X-ray data with the TMAP model of the static atmospheres of Rauch et al. (2010), we found that the WD in Nova SMC 2016 reached a peak $T_{\text {eff }}$ between 850,000 and $900,000 \mathrm{~K}$. In the nova models, such a temperature indicates a WD of $\simeq 1.25 M_{\odot}$, a conclusion also reached by Aydi et al. (2018). We also found absorption features of intermediate-mass elements and magnesium that are not observed in V4743 Sgr, a nova observed at a similar temperature in the SSS phase that is thought to have occurred on a carbon-oxygen WD. This may imply that the WD here is oxygen-neon, but more sophisticated modeling is still needed.

These observations highlight the potential of X-ray gratings to detect very interesting physical characteristics of nova WDs. 
The SSS source is the only phase in which we can observe nuclear burning very close to the stellar surface. Our spectra cannot be perfectly fitted with the published atmospheric grids, but the static models do match the absorption edges and the overall structure of the absorption features. By experimenting with the WT model of van Rossum (2012), we found evidence that the residual wind from this nova occurs in a different mode than the winds of massive $\mathrm{O}$ and $\mathrm{B}$ stars assumed as a template for this model and/or that it should involve a small amount of outflowing mass. In fact, assuming physics similar to the massive stars' winds, the model fails to account for the sharp absorption edges, deep absorption features, and drop in the flux of the softer continuum that are observed in this nova.

The fit with the TMAP atmospheric model fine-tuned for the abundances of V4743 Sgr (Rauch et al. 2010) reproduces the deep nitrogen absorption features for day 39 with $\left[\mathrm{N} / \mathrm{N}_{\odot}\right]=$ 1.803 and $\left[\mathrm{C} / \mathrm{C}_{\odot}\right]=-1.513$. This implies a very large $\mathrm{N} / \mathrm{C}$ ratio, as expected for the ashes of CNO burning, and likely indicates that the burning is not occurring in freshly accreted material after thermonuclear runaway, which would dilute the abundances and, on the other hand, probably also not in coredredged matter (which would have a different composition and be richer in carbon). A highly enhanced nitrogen-to-carbon $(\mathrm{N} / \mathrm{C})$ ratio, as we found, is typical only of the atmosphere on an envelope that has been accreted and, in large part, already burned. Thus, this nova is probably burning in a retained portion of the previously accreted and unejected envelope, leaving the possibility open that the WD may be increasing in mass over its secular evolution. The TMAP fit for the spectra on days 75 and 88 indicates a decrease of the high $\mathrm{N} / \mathrm{C}$ ratio, possibly indicating that accretion of material from the secondary has started again.

We do not have any atmospheric model with abundances appropriate for a neon-oxygen WD yet, but because of the spectral features of magnesium and of intermediate atomic mass elements (calcium, argon, and tentatively sulfur) and by comparison with other novae, we detect possible evidence of an enhancement of the above elements, as expected for the peculiar nucleosynthesis on oxygen-neon WDs because of the neon-sodium and magnesium-aluminum cycles mentioned above. The abundances will be investigated with a fine-tuned model by some of us (T. Rauch et al. 2018, in preparation).

The X-ray grating spectra of novae yield copious physical information, but we are exploring "virgin territory" and are still developing the tools to achieve a thorough understanding. By identifying prominent spectral lines that indicate enhanced abundances and matching them with atmospheric models, we plan to obtain the physical classification of the underlying WD.

As noted by Ness et al. (2011) for the spectra of three Galactic novae, we could not yet identify all of the features in these intricate spectra. Some of these unidentified lines are common to different novae. It is likely that we will ask the producers of atomic data to develop a database to account for these lines; however, since we do not have a fine-tuned atmospheric model yet, we cannot completely rule out that some unidentified features are produced by photoionization or even collisional ionization far from the WD, namely in the ejecta, at different velocities than the system of atmospheric lines.

The X-ray gratings' spectra of N SMC 2016 also show the potential of the grating spectra of luminous novae in the
Magellanic Clouds to yield the chemical composition of the ISM along their lines of sight, using the novae as lamps.

Moreover, the long exposures needed to obtain grating spectra have yielded new information on the short-term light curves of many novae, on timescales of hours. In this nova, we detected a large irregular variability and red noise, but no clear periodicities. Quite surprisingly, we did not find evidence that the flux variability was associated with variable absorption. We do not think that this necessarily means that the variability is intrinsic to the WD atmosphere; we hypothesize that intermittent bursts of mass ejection caused the variability, with ejection of material that was completely optically thick to the SSS radiation but was not ejected in a spherically symmetric manner, hiding a portion of the WD. This phenomenon would explain the non-absorption-dependent variability, and if this is the correct explanation for it, the outflow of matter from the nova must be variable or intermittent on a timescale of hours. So far, intermittent mass ejection, although on timescales of weeks, has only been invoked to explain the light curve of T Pyx (Chomiuk et al. 2014). However, we also note that the complete disappearance of the WD, for hours at the end of one X-ray observation of V4743 Sgr (Ness et al. 2003), seems to be best explained by a new "burst" of ejected mass that was optically thick to the supersoft X-rays.

Since the nova has returned to a quiescent status and the nova shell is expanding away from the WD, while accretion should have resumed, in the near future we hope to obtain an optical spectrum of the central accreting source and measure whether systemic velocity is indeed compatible with SMC membership. This will confirm that N SMC 2016 exceeded Eddington luminosity for more than three months, probably close to half a year, and belongs to a rare group of superluminous novae.

Facilities: Chandra, XMM-Newton.

Software: XSPEC (v12.6.0 Arnaud 1996), CIAO (v4.9; Fruscione et al. 2006), XMM-SAS v16.1.0.

\section{ORCID iDs}

M. Orio (iD https://orcid.org/0000-0003-1563-9803

M. Della Valle (i) https://orcid.org/0000-0003-3142-5020

M. Hernanz (ib https://orcid.org/0000-0002-8651-7910

K. L. Page (ib https://orcid.org/0000-0001-5624-2613

S. Starrfield (ib https://orcid.org/0000-0002-1359-6312

R. E. Williams (1D https://orcid.org/0000-0002-3742-8460

P. Zemko (i) https://orcid.org/0000-0001-8408-0420

\section{References}

Arnaud, K. A. 1996, in ASP Conf. Ser. 101, Astronomical Data Analysis Software and Systems V, ed. G. H. Jacoby \& J. Barnes (San Francisco, CA: ASP), 17

Aydi, E., Page, K. L., Kuin, N. P. M., et al. 2018, MNRAS, 474, 2679

Bailer-Jones, C. A. L., Rybizki, J., Fouesneau, M., Mantelet, G., \& Andrae, R. 2018, arXiv:1804.10121

Bath, G. T., \& Shaviv, G. 1976, MNRAS, 175, 305

Burrows, D. N., Hill, J. E., Nousek, J. A., et al. 2005, SSRv, 120, 165

Cash, W. 1979, ApJ, 228, 939

Chomiuk, L., Nelson, T., Mukai, K., et al. 2014, ApJ, 788, 130

della Valle, M. 1991, A\&A, 252, L9

Dobrotka, A., Ness, J.-U., Mineshige, S., \& Nucita, A. A. 2017, MNRAS, 468, 1183

Fruscione, A., McDowell, J. C., Allen, G. E., et al. 2006, Proc. SPIE, 6270, $62701 \mathrm{~V}$

Gatuzz, E., \& Churazov, E. 2018, MNRAS, 474, 696 
Gatuzz, E., García, J., Kallman, T. R., Mendoza, C., \& Gorczyca, T. W. 2015, ApJ, 800, 29

Gatuzz, E., García, J. A., Kallman, T. R., \& Mendoza, C. 2016, A\&A, 588, A111

Gatuzz, E., Ness, J.-U., Gorczyca, T. W., et al. 2018, MNRAS, 479, 2457

Hachisu, I., Kato, M., \& Luna, G. J. M. 2007, ApJL, 659, L153

Haschke, R., Grebel, E. K., \& Duffau, S. 2012, AJ, 144, 107

Henze, M., Pietsch, W., Haberl, F., et al. 2014, A\&A, 563, A2

Hillman, Y., Prialnik, D., Kovetz, A., \& Shara, M. M. 2016, ApJ, 819, 168

Jablonski, F., \& Oliveira, A. 2016, ATel, 9684

José, J., \& Hernanz, M. 1998, ApJ, 494, 680

Kelly, K. J., Iliadis, C., Downen, L., José, J., \& Champagne, A. 2013, ApJ, 777,130

Lanz, T., Telis, G. A., Audard, M., et al. 2005, ApJ, 619, 517

Lipunov, V., Podesta, R., Levato, H., et al. 2016, ATel, 9631

Luri, X., Brown, A. G. A., Sarro, L. M., et al. 2018, arXiv:1804.09376

Nelson, T., Orio, M., Cassinelli, J. P., et al. 2008, ApJ, 673, 1067

Ness, J.-U., Starrfield, S., Burwitz, V., et al. 2003, ApJL, 594, L127

Ness, J.-U., Starrfield, S., Beardmore, A. P., et al. 2007, ApJ, 665, 1334

Ness, J.-U., Osborne, J. P., Dobrotka, A., et al. 2011, ApJ, 733, 70

Nicastro, F., Senatore, F., Gupta, A., et al. 2016a, MNRAS, 457, 676

Nicastro, F., Senatore, F., Gupta, A., et al. 2016b, MNRAS, 458, L123

Orio, M. 2012, BASI, 40, 333

Orio, M., Balman, S., della Valle, M., Gallagher, J., \& Oegelman, H. 1996, ApJ, 466, 410
Orio, M., Parmar, A., Benjamin, R., et al. 2001, MNRAS, 326, L13

Orio, M., Behar, E., Gallagher, J., et al. 2013, MNRAS, 429, 1342

Peretz, U., Orio, M., Behar, E., et al. 2016, ApJ, 829, 2

Poggiani, R. 2018, arXiv:1803.11529

Predehl, P., \& Schmitt, J. H. M. M. 1995, A\&A, 293, 889

Prialnik, D., \& Kovetz, A. 2005, in AIP Conf. Ser. 797, Interacting Binaries: Accretion, Evolution, and Outcomes, ed. L. Burderi et al. (Melville, NY: AIP), 319

Rauch, T., Orio, M., Gonzales-Riestra, R., et al. 2010, ApJ, 717, 363

Scargle, J. D. 1982, ApJ, 263, 835

Schwarz, G. J., Shore, S. N., Starrfield, S., et al. 2001, MNRAS, 320, 103

Scowcroft, V., Freedman, W. L., Madore, B. F., et al. 2016, ApJ, 816, 49

Shafter, A. W., Rau, A., Quimby, R. M., et al. 2009, ApJ, 690, 1148

Shumkov, V., Lipunov, V., Podesta, R., et al. 2016, ATel, 9621

Starrfield, S., Iliadis, C., \& Hix, W. R. 2016, PASP, 128, 051001

Starrfield, S., Iliadis, C., Hix, W. R., Timmes, F. X., \& Sparks, W. M. 2009, ApJ, 692, 1532

Starrfield, S., Timmes, F. X., Iliadis, C., et al. 2012, BaltA, 21, 76

Strope, R. J., Schaefer, B. E., \& Henden, A. A. 2010, AJ, 140, 34

Timmer, J., \& Koenig, M. 1995, A\&A, 300, 707

Tofflemire, B. M., Orio, M., Page, K. L., et al. 2013, ApJ, 779, 22

van Rossum, D. R. 2012, ApJ, 756, 43

Warner, B. 2003, Cataclysmic Variable Stars (Cambridge: Cambridge Univ. Press)

Wilms, J., Allen, A., \& McCray, R. 2000, ApJ, 542, 914

Wolf, W. M., Bildsten, L., Brooks, J., \& Paxton, B. 2013, ApJ, 777, 136 